\title{
Herbáceas Aquáticas em Igapós de Água Preta Dentro e Fora de Unidades de Conservação no Estado do Amazonas
}

\author{
Aline Lopes ${ }^{1,2}$, Luciana Carvalho Crema ${ }^{1,3}$, Layon Oreste Demarchi ${ }^{1}$, Aurélia Bentes Ferreira ${ }^{1}$, Ivone Santiago ${ }^{1}$, \\ Eduardo Antonio Ríos Villamizar ${ }^{1} \&$ Maria Teresa Fernandez Piedade ${ }^{1}$
}

Recebido em 31/01/2018 - Aceito em 09/10/2018

\begin{abstract}
RESUMO - As herbáceas aquáticas são indicadoras da composição nutricional dos corpos de água amazônicos e de suas áreas alagáveis, pois sua riqueza e abundância refletem as características físicas e químicas da bacia onde estão inseridas. Elas possuem diversas funções ecológicas, como proporcionar abrigo e alimento para organismos aquáticos e terrestres. Neste estudo, foi analisada a riqueza, diversidade e composição de espécies de herbáceas aquáticas e sua distribuição em nove igapós de água preta na Amazônia brasileira, dentro e fora de unidades de conservação (UCs). Os resultados foram obtidos por meio de inventários realizados entre os anos de 2009 e 2017, nos rios Negro, Jaú, Aracá, Cuiuni, Uatumã e Abacate. Foram amostradas 148 parcelas retangulares de $100 \times 1 \mathrm{~m}$, distribuídas em nove pontos amostrais (6 a 29 parcelas por ponto). O levantamento florístico revelou 174 espécies, distribuídas em 99 gêneros e 46 famílias botânicas, predominando as famílias Cyperaceae (43 ssp.), Poaceae (33 ssp.) e Marantaceae (10 ssp.). As áreas apresentaram baixa similaridade florística, sendo que nenhuma espécie ocorreu em todas as áreas amostradas. As espécies Montrichardia arborescens (L.) Schott (Araceae) e Scleria secans (L.) Urb (Cyperaceae) foram as mais frequentes, ocorrendo em seis das nove áreas amostradas. Apenas 49,2\% das espécies de herbáceas aquáticas amostradas estão presentes nas UCs inventariadas, indicando que estratégias de conservação que envolvam mais áreas de igapó de águas pretas precisam ser consideradas para a proteção desse importante grupo de plantas.
\end{abstract}

Palavras-chave: Áreas úmidas; macrófitas aquáticas; similaridade florística.

ABSTRACT - Aquatic Herbaceous in Black Water Igapós Inside and Outside Conservation Units in the State of Amazonas. Aquatic herbaceous plants are indicative of the nutritional composition in Amazonian water bodies and their wetlands, because their richness and abundance reflect the physical and chemical characteristics of the basin where they are inserted. They have several ecological functions, such as providing shelter and food for aquatic and terrestrial organisms. In this study the richness and composition of aquatic herbaceous species and their distribution were analyzed in nine black water floodplains (igapó) in Brazilian Amazonia, inside and outside protected areas (PAs). The results were obtained through inventories carried out between the years 2009 and 2017 in the Negro, Jaú, Aracá, Cuiuni, Uatumã and Abacate rivers. We used 148 rectangular plots of $100 \times 1 \mathrm{~m}$ at nine sampling points (ranging from 6 to 29 plots

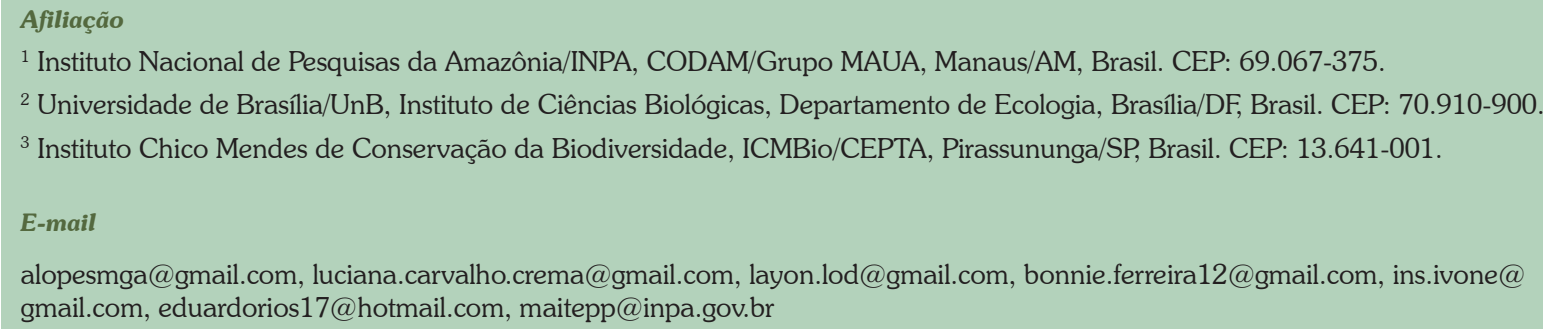


per point). The floristic inventory sampled 174 species, distributed in 99 genera and 46 botanical families, predominantly Cyperaceae (43 ssp.), Poaceae (33 ssp.) and Marantaceae (10 ssp.). The areas showed low floristic similarity, and no species occurred in all sampled places. The species Montrichardia arborescens (L.) Schott (Araceae) and Scleria secans (L.) Urb (Cyperaceae) were the most frequent occurring in six of the nine sampled areas. Only $49.2 \%$ of the aquatic herbaceous species are present in the inventoried PAs showing that conservation strategies involving more areas of black water floodplains need to be considered for the protection of this important plant group.

Keywords: Aquatic macrophytes; floristic similarity; Wetlands.

RESUMEN - Herbáceas Acuáticas en Aguas Negras de Igapós Dentro y Fuera de las Unidades de Conservación en el Estado de Amazonas. Las herbáceas acuáticas son indicadoras de la composición nutricional de los cuerpos de agua amazónicos y de sus áreas inundables, ya que su riqueza y su abundancia reflejan las características físicas y químicas de la cuenca donde están situadas. Ellas poseen diversas funciones ecológicas, como proporcionar abrigo y alimento para organismos acuáticos y terrestres. En este estudio se analizó la riqueza y composición de especies de herbáceas acuáticas y su distribución en nueve humedales de agua negra (igapós) en la Amazonía brasileña, dentro y fuera de unidades de conservación (UCs). Los resultados fueron obtenidos por medio de inventarios realizados entre los años 2009 y 2017 en los ríos Negro, Jaú, Aracá, Cuiuni, Uatumã y Abacate. Se tomaron muestras en 148 parcelas rectangulares de $100 \times 1$ m distribuidas en nueve puntos de muestreo ( 6 a 29 parcelas por punto). El levantamiento florístico reveló 174 especies, distribuidas en 99 géneros y 46 familias botánicas, predominando las familias Cyperaceae (43 ssp.), Poaceae (33 ssp.) y Marantaceae (10 ssp.). Las áreas presentaron baja similitud florística y ninguna especie ocurrió en todas las áreas muestreadas. Las especies Montrichardia arborescens (L.) Schott (Araceae) y Scleria secans (L.) Urb (Cyperaceae) fueron las más frecuentes y ocurrieron en seis de las nueve áreas estudiadas. Solamente 49,2\% de las especies de herbáceas acuáticas muestreadas están presentes en UCs inventariadas, indicando que las estrategias de conservación que involucren más áreas de igapó de aguas negras necesitan ser consideradas para la protección de ese importante grupo de plantas.

Palabras clave: Macrófitas acuáticas; similitud florística; humedales.

\section{Introdução}

A existência de ecossistemas inundáveis está relacionada à dinâmica da bacia Amazônica, que é submetida a ciclos de inundações periódicas (Junk et al. 1989), devido às flutuações dos níveis das águas dos rios, cuja amplitude pode alcançar de 10 a $14 \mathrm{~m}$ entre o pico das cheias (fase aquática) e o pico das secas (fase terrestre). Isso corresponde a períodos de alagamento de $50 \mathrm{a}$ 279 dias, conforme a altitude da planície inundável considerada (Junk 1980), o que faz com que, durante o ano, as plantas precisem alternar o seu ciclo de vida conforme essa dinâmica. O pulso de inundação exerce uma forte influência sobre a vegetação herbácea desses ecossistemas (Piedade et al. 2010).

Com base na tipologia das águas de Sioli (1985) e na análise de suas florestas associadas (Prance 1979, 1980), dois grandes grupos de áreas alagáveis se destacam na região: as várzeas e os igapós. As várzeas são periodicamente inundadas por rios de águas brancas, dominam a planície amazônica, ocorrem em formações do Quaternário recente e são constituídas por sedimentos ricos em nutrientes e com elevada dinâmica hidrogeomorfológica (Prance 1979, Junk et al. 2012). Possuem fertilidade relativamente elevada, que se reflete no crescimento rápido da vegetação herbácea (Piedade et al. 1991; Junk \& Piedade 1993, 1997) e na acelerada ciclagem de nutrientes (Junk \& Furch 1991; Furch \& Junk 1992, 1997). Os ambientes de igapó, por sua vez, são áreas alagáveis por rios de águas pretas ou claras, que drenam formações mais antigas do período Terciário; apresentam níveis de nutrientes mais baixos que as várzeas e $\mathrm{pH}$ ácido a ligeiramente ácido (Fittkau et al. 1975, Sioli 1984, Junk et al. 2015).

Estudos de análise da composição de herbáceas aquáticas nos igapós ainda são escassos (Piedade et al. 2010). Sabe-se, contudo, que nos igapós de águas pretas há predominância de espécies vegetais flutuantes ou emersas (Ferreira 2000), com baixa similaridade na composição 
florística entre diferentes áreas (Lopes et al. 2014). Já nos igapós de águas claras, apesar da presença de espécies emergentes, é notória a presença de plantas submersas, favorecidas pela elevada transparência da água (Crema 2017). Mesmo havendo uma grande variação entre os ambientes, as várzeas e os igapós de águas claras apresentam composição florística diferente e riqueza de herbáceas aquáticas maiores do que as encontradas nos igapós de águas pretas; porém, o baixo esforço amostral realizado nesse tipo de igapó ainda impede maiores conclusões (Piedade et al. 2010, Lopes et al. 2014).

As herbáceas aquáticas são de extrema importância como habitat para a fauna aquática $e$ terrestre, devido à grande produção de biomassa e à ampla extensão que ocupam (Piedade \& Junk 2000, Petry et al. 2003). Proporcionam local adequado para o desenvolvimento de vários organismos invertebrados e vertebrados, pois suas raízes servem de substrato para a deposição de ovos e refúgio para o zooplâncton (Murphy 1988, Esteves 1998), bem como para juvenis de peixes que podem evitar a predação, encontrando abrigo e alimento entre os talos, raízes e folhas (Piedade et al. 2005). Quanto a mamíferos, o peixe-boi da Amazônia (Trichehus inunguis) e a capivara (Hydrochoerus hydrochaeris) são os mais importantes consumidores de herbáceas aquáticas (Best 1984, Junk \& Silva 1997, Piedade et al. 2010, Crema et al. 2019). Independente da tipologia das águas que inundam o igapó, seus lagos são habitat essenciais na planície de inundação, já que, mesmo durante períodos de seca, muitos deles se mantêm com coluna de água (Fernandes et al. 2009) e são ricos em recursos alimentares (Junk 1980).

No Brasil, a conservação in situ da biodiversidade é a realizada em maior escala (Hassler 2005) nas unidades de conservação (UCs) e terras indígenas. Esses territórios objetivam contribuir para a conservação de espécies biológicas e recursos genéticos e culturais, proteger as espécies ameaçadas de extinção, bem como contribuir para a preservação e a restauração da diversidade de ecossistemas naturais, dentre outros (Brasil 2000). Dessa forma, sobretudo as UCs teriam o papel principal para conservação de espécies, fossem elas aquáticas ou terrestres. A Amazônia possui 61.992 .285 ha pertencentes a UCs federais, o que equivale a 7,27\% do território nacional (ICMBio 2019). No estado do Amazonas, as UCs correspondem a 18.907.378ha (SEMA 2018) e, no caso da bacia do rio Negro, a área estimada apenas para UCs federais é de cerca de 7.740.00ha (ICMBio 2018).

Dentro das UCs que contemplam áreas de igapós, poucos estudos foram realizados com herbáceas aquáticas (Lopes et al. 2014), porém eles apontam para uma baixa similaridade florística, mesmo no nível de gênero das herbáceas aquáticas em igapós de águas pretas, sugerindo que a conservação desse grupo de plantas precisa ser pensada considerando diversas áreas de UCs dentro da bacia. Com o objetivo de ampliar o conhecimento a respeito das espécies de herbáceas aquáticas de igapós de águas pretas, no presente estudo foi analisada a composição florística de nove áreas de igapó na bacia Amazônica, quatro delas em UCs e cinco em áreas fora de UCs.

\section{Materiais e métodos}

Foram inventariadas, dentro e fora de UCs localizadas no estado do Amazonas, 148 parcelas retangulares de $100 \mathrm{~m}$ de comprimento x $1 \mathrm{~m}$ de largura, com $500 \mathrm{~m}$ de distância entre si e dispostas nas margens dos rios: Aracá (Barcelos - fora de UC); Abacate (São Sebastião do Uatumã - Reserva de Desenvolvimento Sustentável do Uatumã); Negro (Novo Airão - Parque Nacional de Anavilhanas); Negro (Arquipélago de Mariuá - fora de UC); Negro (Barcelos - fora de UC); Negro (Santa Isabel do rio Negro - fora de UC); Cuiuni (Barcelos - fora de UC), Jaú (Novo Airão - Parque Nacional do Jaú) e Uatumã (Presidente Figueiredo - Reserva de Desenvolvimento Sustentável do Uatumã), todos pertencentes à bacia do rio Negro, com exceção dos rios Uatumã e Abacate, que fazem parte da bacia do rio Amazonas (Figura 1). O número de parcelas foi otimizado com base na curva de suficiência amostral, variando entre 6 a 29 parcelas para cada local de coleta (Tabela 1). 
As coletas foram realizadas entre 2009 e 2017. Foram coletadas plantas herbáceas aquáticas, semi-aquáticas e terrestres, conforme a classificação de Junk \& Piedade (1993), presentes nas planícies de inundação dos rios selecionados. Todas as espécies inventariadas foram fotografadas ou coletadas para posterior identificação. As identificações foram feitas até o menor nível taxonômico possível, por meio de chaves taxonômicas de famílias e gêneros do Neotropikey (kew.org/science/tropamerica/neotropikey.htm), e comparadas com as exsicatas identificadas por especialistas, e depositadas nos herbários do Instituto Nacional de Pesquisas da Amazônia (INPA) e do Grupo Ecologia, Monitoramento e Uso Sustentável de Áreas Úmidas (MAUA), em Manaus. A nomenclatura utilizada está de acordo com o APG IV (2016) e foi conferida conforme o projeto Reflora (Flora do Brasil 2020). Todas as herbáceas coletadas foram classificadas de acordo com seu hábito, adaptado de Junk (1986) e Piedade et al. (2018). A distribuição das espécies foi determinada pelo cruzamento de informações entre os projetos Reflora (Flora do Brasil 2020), Tropicos (Tropicos.org) e GBIF (Global Biodiversity Information Facility).

Para caracterização geral e descrição das áreas de coleta, foram tomadas em campo, com aparelhos portáteis, concomitantemente aos inventários florísticos, medidas de $\mathrm{pH}$, condutividade elétrica $\left(\mu \mathrm{S} \mathrm{cm}^{-1}\right)$, temperatura $\left({ }^{\circ} \mathrm{C}\right)$ e transparência (disco de Secchi, $\mathrm{cm}$ ) da água em cada umas das 148 parcelas (Tabela 1 ).
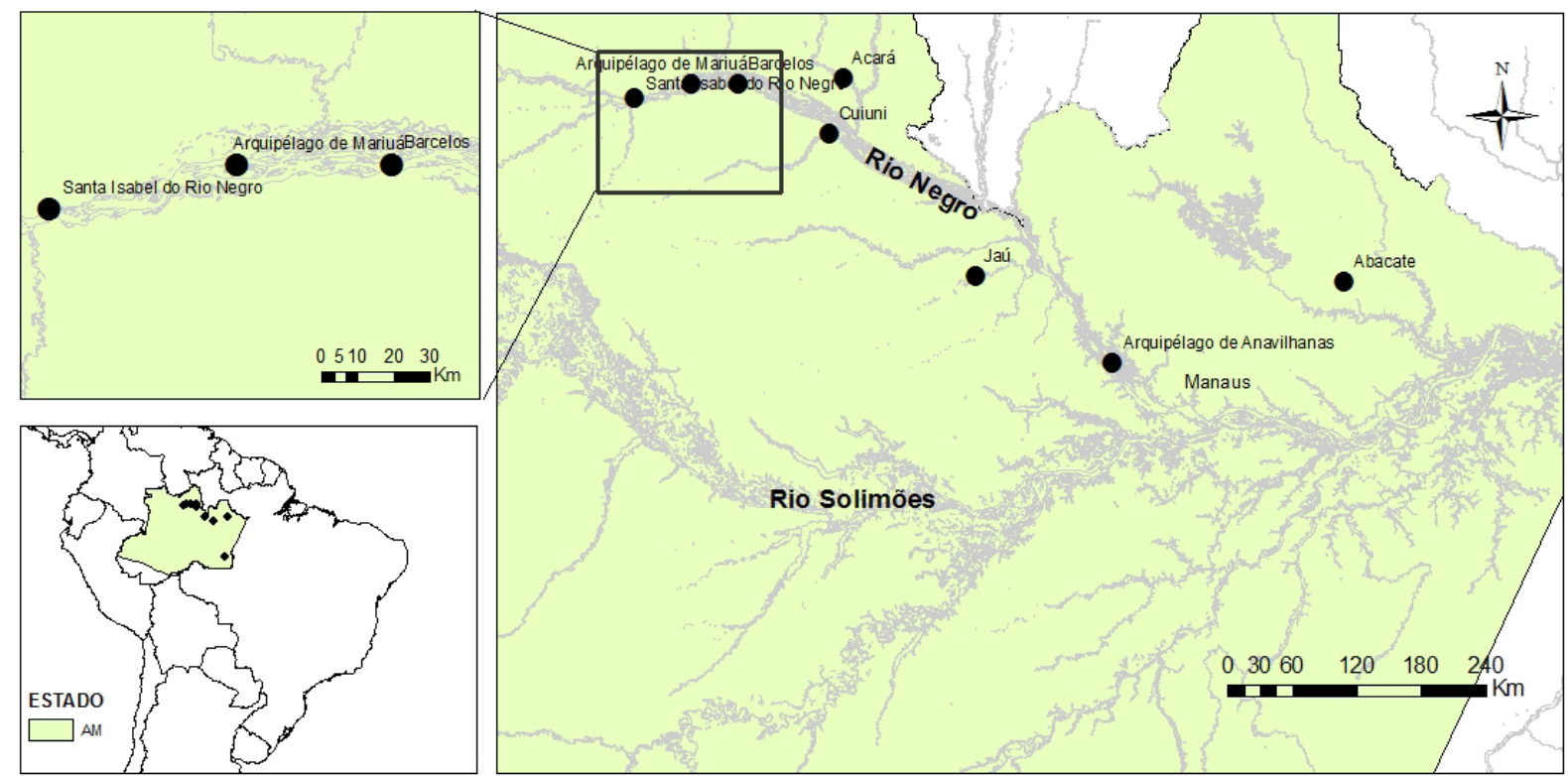

Figura 1 - Área de estudo, igapós dos rios Cuiuni, Uatumã, Negro (PARNA de Anavilhanas, Arquipélago de Mariuá, Santa Isabel do Rio Negro e Barcelos), Jaú, Abacate e Aracá, no estado do Amazonas.

Figure 1 - Study área, black-water foodplains "igapós" of rivers: Cuiuni, Uatumã, Negro (PARNA de Anavilhanas, Arquipélago de Mariuá, Santa Isabel do Rio Negro and Barcelos), Jaú, Abacate and Aracá, in Amazonas state.

\section{Análise de dados}

Para avaliar a diversidade alfa nas comunidades, foi utilizado o índice de Simpson (D), que é pouco influenciado por espécies raras (Magurran 1988). A diversidade beta foi avaliada pelo índice de Whittaker (b), que mede a mudança ou taxa de substituição na composição de espécies de um local para outro (Whittaker 1960). Esse índice varia de 0, quando duas amostras não apresentam nenhuma diferença na composição de espécies, a 2, quando essa diferença é máxima, 
sendo calculado pela fórmula: $\beta=(\mathrm{c} / \mathrm{a}) 3 / 41$, onde: $\mathrm{c}=$ total de espécies nas parcelas amostradas; $\mathrm{a}=$ média do número de espécies das parcelas amostradas. A similaridade florística foi analisada utilizando a técnica de ordenação indireta por meio de NMDS (Escalonamento Multidimensional não Paramétrico) com os dados de presença e ausência das espécies em cada um dos igapós. Como medida de distância foi utilizado o índice de Jaccard. O NMDS reduz a composição da comunidade em duas dimensões, não assume relações lineares entre variáveis e não depende de um índice de associação específico, frequentemente resumindo mais informação em poucos vetores do que outros métodos de ordenação (Manly 2004). Para mensurar a qualidade do ajuste, foi utilizada a medida stress, que indica a proporção da variação das distâncias originais em relação às distâncias preditas pelo NMDS. A similaridade florística entre as áreas foi também medida pela análise de agrupamento aplicado para a distância de Jaccard, utilizando-se o método de ligação UPGMA (Unweight Pair-Group Method Average) (Sneath \& Sokal 1973), sendo avaliado o coeficiente de correlação cofenético $(\mathrm{r})$. Todas essas análises foram realizadas pelo programa PAST 3.0 (Hammer et al. 2001).

\section{Resultados e discussão}

Adistribuição e o crescimento da vegetação aquática têm sido tradicionalmente compreendidos em termos de respostas ecofisiológicas das plantas às condições ambientais locais, tais como luz, temperatura, disponibilidade de nutrientes, $\mathrm{pH}$, salinidade, velocidade da água e variação do nível de água, (Barendregt \& Bio 2003, Neiff \& Neiff 2003, Henry-Silva \& Camargo 2005, Cancian et al. 2009, Lopes et al. 2016, Lopes et al. 2017). Todas as áreas inventariadas apresentaram características hidroquímicas típicas de igapó de águas pretas (Prance 1980), com baixos valores de $\mathrm{pH}$, e condutividade elétrica e alta transparência da água (Tabela 1). A condutividade elétrica média variou entre $7,17 \mu \mathrm{S} / \mathrm{cm}$, no rio Cuiuni, e 17,85 $\mu \mathrm{S} / \mathrm{cm}$, no rio Abacate. Os valores médios de pH variaram entre 3,64, no rio Jaú, e 5,46, no rio Abacate. Já os valores médios de transparência variaram entre $0,90 \mathrm{~m}$, no Jaú, e $2,76 \mathrm{~m}$, no Uatumã. O baixo valor de $\mathrm{pH}$ das águas dos igapós de águas pretas, juntamente com a baixa concentração de nutrientes, são os principais limitantes para a sobrevivência das plantas aquáticas, fazendo com que a diversidade de espécies seja bastante reduzida nessas áreas (Piedade et al. 2010).

Tabela 1 - Número de parcelas, riqueza de espécies, diversidade alfa de Simpson e dados físicos e químicos da água dos rios inventariados na bacia Amazônica.

Table 1 - Number of plots, species richness, Simpson alpha diversity and physical and chemical data of water from the rivers inventoried in the Amazon basin.

\begin{tabular}{|l|c|c|c|c|c|c|c|}
\hline \multicolumn{1}{|c|}{ Rios } & No. parcelas & Riqueza & Simpson & $\begin{array}{c}\text { Secchi } \\
(\mathbf{m})\end{array}$ & $\begin{array}{c}\text { Temperatura } \\
\left({ }^{\circ} \mathbf{C}\right)\end{array}$ & $\begin{array}{c}\text { Condutividade } \\
(\boldsymbol{\mu S} / \mathbf{c m})\end{array}$ & $\mathbf{p H}$ \\
\hline Aracá & 18 & 27 & 0,96 & 1,22 & 29,00 & 16,60 & 3,77 \\
\hline Cuiuni & 29 & 23 & 0,96 & 1,33 & 27,17 & 7,17 & 3,82 \\
\hline Jaú & 16 & 24 & 0,96 & 0,90 & 27,10 & 8,60 & 3,64 \\
\hline Negro (Barcelos) & 18 & 33 & 0,97 & 1,00 & 30,90 & 11,30 & 4,28 \\
\hline Uatumã & 7 & 27 & 0,95 & 2,76 & 30,34 & 7,83 & 5,34 \\
\hline Negro (PARNA de Anavilhanas) & 12 & 22 & 0,98 & 0,99 & 29,54 & 8,82 & 5,07 \\
\hline Negro (Santa Isabel do Rio Negro) & 18 & 48 & 0,96 & ND & ND & ND & 4,631 \\
\hline Negro (Arquipélago de Mariuá) & 24 & 27 & 0,96 & ND & ND & $14,50^{1}$ & $4,42^{1}$ \\
\hline Abacate & 6 & 24 & 0,96 & 1,53 & 28,05 & 17,85 & 5,46 \\
\hline
\end{tabular}

${ }^{1}$ Küchler et al. 2000; ND = não determinado. 
Foram analisados 255 registros de herbáceas aquáticas, compreendendo os hábitos de vida aquática, semi-aquática e terrestre, num total de 174 espécies, distribuídas em 99 gêneros e 46 famílias. Esse registro é um grande incremento no número de espécies de herbáceas de água preta na Amazônia, cuja última estimativa era em torno de 75 espécies (Lopes et al. 2014). O maior número de espécies de herbáceas aquáticas foi encontrado nas margens do rio Negro, com 48 espécies em Santa Isabel do Rio Negro; 33 espécies em Barcelos; e 27 espécies no Arquipélago Mariuá (Tabela 1). O maior número de espécies na planície de inundação do rio Negro em comparação aos seus tributários pode ser reflexo de uma das formas predominantes de dispersão das plantas aquáticas, que é pela água (Thomaz 2002), ou seja, seguindo o sentido da correnteza.

A maioria das espécies inventariadas nos igapós foi considerada erva com 147 espécies; seguidas pelas trepadeiras, com 17 espécies; ervas/subarbustos, seis; e ervas/trepadeiras, quatro. Apenas 18 espécies foram consideradas estritamente aquáticas; 30 puderam ser encontradas em ambiente palustre ou aquático; e 126 terrestres ou palustres (aproximadamente $72,4 \%$ ). A maior parte das espécies foi encontrada às margens dos rios e lagos, sendo consideradas anfíbias ou terrestres (125), seguidas das anfíbias (19) e das anfíbias/emergentes (6) (Tabela 2). A predominância de espécies terrestres e palustres também ocorre em várzeas alagadas por água branca. Junk \& Piedade (1993) estimaram em $85 \%$ o número de espécies terrestres na várzea próxima a Manaus, contra $8 \%$ de espécies aquáticas. As espécies palustres e terrestres são principalmente anuais e possuem seu ciclo de vida sincronizado com o pulso de inundação, possuindo propágulos ou sementes resistentes à inundação (Piedade et al. 2010). Dessa forma, a conservação das margens dos rios e a manutenção dos ciclos de cheia e seca são essenciais para a permanência dessa vegetação, tanto nas várzeas quanto nos igapós. Embora o número de espécies de herbáceas aquáticas nos igapós de água preta seja menor que nas várzeas amazônicas, que contam com aproximadamente 400 espécies (Junk \& Piedade 1993, Piedade et al. 2010), o presente estudo indicou para igapós de águas pretas um valor bem superior (174 espécies) ao anteriormente registrado (75 espécies, Lopes et al. 2014). Nesses igapós, onde a oferta de recursos é mais escassa, essas plantas podem desempenhar um papel fundamental como fonte de alimento para peixes e outros herbívoros, como o peixe-boi-daAmazônia. Para esse mamífero aquático ameaçado de extinção, a presença $e$ a abundância dessas plantas podem ser determinantes para a permanência ou a migração da espécie para diferentes áreas (Arraut et al. 2010, Crema et al. 2019), sejam elas protegidas ou não.

Tabela 2 - Hábito das herbáceas aquáticas inventariadas nos igapós dos rios: Cuiuni, Uatumã, Negro (PARNA de Anavilhanas), Jaú, Negro (Arquipélago de Mariuá), Abacate, Aracá, Negro (Barcelos), Negro (Santa Isabel do Rio Negro) na bacia Amazônica.

Table 2 - Habitat of aquatic herbaceous plants recorded in black-water floodplains of rivers: Cuiuni, Uatumã, Negro (PARNA de Anavilhanas), Jaú, Negro (Archipelago Mariuá), Abacate, Aracá, Negro (Barcelos), Negro (Santa Isabel do Rio Negro) in the Amazon basin.

\begin{tabular}{|l|c|}
\hline \multicolumn{1}{c|}{ Hábito } & No de espécies \\
\hline Anfíbia/terrestre & 125 \\
\hline Anfibia & 19 \\
\hline Anfíbia/emergente & 6 \\
\hline Emergente/flutuante livre emersa & 5 \\
\hline Fixa com folhas flutuantes & 5 \\
\hline Flutuante livre emersa & 4 \\
\hline Submersa fixa & 3 \\
\hline Submersa fixa/emergente & 3 \\
\hline Emergente & 2 \\
\hline Anfibia/flutuante livre submersa & 1 \\
\hline Flutuante livre submersa & 1 \\
\hline
\end{tabular}


As famílias que apresentaram o maior número de espécies foram Cyperaceae (44 espécies), Poaceae (33 espécies) e Marantaceae (10 espécies). Sendo os gêneros Rhynchospora (Cyperaceae), com nove espécies; Paspalum (Poaceae), com oito espécies; e Cyperus (Cyperaceae), com sete espécies, os mais comuns. As famílias Cyperaceae e Poaceae aparecem em outros inventários em áreas alagáveis na Amazônia como as que possuem o maior número de espécies - por exemplo, nos lagos do município de Normandia, em Roraima (RR), a família Cyperaceae também foi citada como o principal táxon (Simões Filho et al. 1997). No lago do Trevo, em Boa Vista/ RR, a família Cyperaceae (19\%) também foi a mais representativa, seguida de Eriocaulaceae (13\%) e Melastomataceae (10\%) (Neves 2007). Em lagos de duas UCs federais ao longo do rio Tapajós, no Pará, Crema (2017) relatou que Poaceae (19\%) e Cyperaceae (23\%) corresponderam a $42 \%$ de todas as plantas amostradas. Já nas áreas de várzea amazônica, ocorre uma inversão de predominância, sendo Poaceae (15,5\%), seguida de Cyperaceae (9,5\%), as famílias com maior número de espécies (Junk e Piedade 1993).

As espécies Montrichardia arborescens (L.) Schott (Araceae) e Scleria secans (L.) Urb. (Cyperaceae) foram as mais frequentes, ocorrendo em seis das nove áreas amostrais. Hypolytrum longifolium (Rich.) Nees (Cyperaceae) ocorreu em quatro áreas; e, dentre as demais espécies, 15 ocorreram em apenas três áreas, 38 ocorreram em duas e 118 ocorreram em apenas uma área amostral, demonstrando a baixa similaridade florística das áreas de igapó estudadas (Tabela 3). Lopes et al. (2014) também encontraram Montrichardia arborescens como a espécie mais comum nesse ecossistema, confirmando a sua preferência por ambientes com baixo $\mathrm{pH}$, como os igapós de água preta (Lopes et al. 2016).

Tabela 3 - Herbáceas aquáticas em igapós de água preta nos rios: 1 - Cuiuni, 2 - Uatumã, 3 - Negro (PARNA de Anavilhanas), 4 - Jaú, 5 - Negro (Arquipélago de Mariuá), 6 - Abacate, 7 - Aracá, 8 - Negro (Barcelos), 9 - Negro (Santa Isabel do Rio Negro) na bacia Amazônica.

Table 3 - Aquatic herbaceous plants in black-water floodplains of rivers: 1 - Cuiuni, 2 - Uatumã, 3 - Negro (PARNA de Anavilhanas), 4 - Jaú, 5 - Negro (Archipelago Mariuá), 6 - Abacate, 7 - Aracá, 8 Negro (Barcelos), 9 - Negro (Santa Isabel do Rio Negro) in the Amazon basin.

\begin{tabular}{|c|c|c|c|c|c|c|c|c|c|c|}
\hline Família & Espécies & 1 & 2 & 3 & 4 & 5 & 6 & 7 & 8 & 9 \\
\hline Acanthaceae & Justicia comata (L.) Lam. & & $\mathrm{x}$ & & & & & & & \\
\hline \multirow{2}{*}{ Alismataceae } & Limnocharis flava (L.) Buchenau & & & & & & & $\mathrm{x}$ & & \\
\hline & Sagittaria guayanensis Kunth & & $\mathrm{x}$ & & & & & & & \\
\hline Amaranthaceae & Alternanthera paronychioides A.St.-Hil. & & $\mathrm{x}$ & & & & & & & \\
\hline \multirow{4}{*}{ Apocynaceae } & Mesechites trifidus (Jacq.) Müll.Arg. & & & $\mathrm{X}$ & & & & & & \\
\hline & Tassadia berteroana (Spreng.) W.D.Stevens & $\mathrm{x}$ & $\mathrm{x}$ & $\mathrm{x}$ & & & & & & \\
\hline & Tassadia propinqua Decne. & & & & & & $\mathrm{x}$ & & & \\
\hline & Tassadia trailiana (Benth.) Fontella & & $\mathrm{x}$ & & $\mathrm{x}$ & & & & & \\
\hline \multirow{7}{*}{ Araceae } & Caladium bicolor (Aiton) Vent. & & & & & & & & & $\mathrm{x}$ \\
\hline & Dieffenbachia elegans A.M.E.Jonker \& Jonker & & & & & $\mathrm{x}$ & & & & $\mathrm{x}$ \\
\hline & Dieffenbachia parvifolia Engl. & & & & & $\mathrm{x}$ & & & & $\mathrm{x}$ \\
\hline & Lemna minuta Kunth & & & $\mathrm{x}$ & & & & & & \\
\hline & Montrichardia arborescens (L.) Schott & $\mathrm{x}$ & $\mathrm{x}$ & & & $\mathrm{x}$ & $\mathrm{x}$ & & $\mathrm{x}$ & $x$ \\
\hline & Philodendron muricatum Willd. ex Schott & & & & & & $\mathrm{x}$ & & & \\
\hline & Urospatha sagittifolia (Rudge) Schott & & & & & & & $\mathrm{x}$ & & \\
\hline \multirow{4}{*}{ Asteraceae } & Erechtites hieracifolius (L.) Raf. ex DC. & & $\mathrm{x}$ & & & & & & & \\
\hline & Mikania micrantha Kunth & & & $\mathrm{x}$ & & & & & & \\
\hline & Unxia camphorata L.f. & & & & & $\mathrm{x}$ & & & & $\mathrm{x}$ \\
\hline & Wedelia calycina Rich. & & $\mathrm{x}$ & & & & & & & \\
\hline \multirow{2}{*}{ Boraginaceae } & Euploca filiformis (Lehm.) J.I.M.Melo \& Semir & & $\mathrm{x}$ & & & & & & & \\
\hline & Heliotropium indicum L. & $\mathrm{x}$ & & & & & & & & \\
\hline
\end{tabular}




\begin{tabular}{|c|c|c|c|c|c|c|c|c|c|c|}
\hline Bromeliaceae & Ananas nanus (L.B.Sm.) L.B.Sm. & & & & & & $\mathrm{X}$ & & & \\
\hline Burmanniaceae & Burmannia polygaloides Schltr. & & & & & & & & & $\mathrm{X}$ \\
\hline Cabombaceae & Cabomba aquatica Aubl. & $\mathrm{x}$ & & & & & & & & \\
\hline Caryophyllaceae & Drymaria cordata (L.) Willd. ex Roem. \& Schult. & & $\mathrm{x}$ & & & & & & & \\
\hline \multirow{2}{*}{ Convolvulaceae } & Ipomoea alba $\mathrm{L}$. & & & $\mathrm{X}$ & & & & & & \\
\hline & Ipomoea rubens Choisy & & & & $\mathrm{X}$ & & & & & \\
\hline Cucurbitaceae & Cayaponia cruegeri (Naudin) Cogn. & & & $\mathrm{X}$ & & & & & & \\
\hline \multirow{41}{*}{ Cyperaceae } & Becquerelia merkeliana Nees & & & & & & $\mathrm{X}$ & $\mathrm{X}$ & & \\
\hline & Bulbostylis junciformis (Kunth) C.B.Clarke & & & & & & & & & $\mathrm{X}$ \\
\hline & Calyptrocarya glomerulata (Brongn.) Urb. & & & & & & $\mathrm{X}$ & & & \\
\hline & Calyptrocarya monocephala Hochst. ex Steud. & & & & & & & $\mathrm{X}$ & & $\mathrm{X}$ \\
\hline & Cyperus aggregatus (Willd.) Endl. & $\mathrm{X}$ & $\mathrm{X}$ & & & & & & & \\
\hline & Cyperus distans L. & & $\mathrm{x}$ & & & & & & & \\
\hline & Cyperus ligularis L. & & & & & & & & & $\mathrm{X}$ \\
\hline & Cyperus luzulae (L.) Retz. & & & & & & & & $\mathrm{X}$ & \\
\hline & Cyperus miliifolius Poepp. \& Kunth & & & & & & & & $\mathrm{X}$ & \\
\hline & Cyperus mutisii (Kunth) Anderson & & & & & & & & & $\mathrm{X}$ \\
\hline & Cyperus odoratus L & $\mathrm{X}$ & & & & & & & & \\
\hline & Diplacrum capitatum (Willd.) Boeckeler & & & & & & & $\mathrm{X}$ & & \\
\hline & Diplacrum guianense (Nees) T.Koyama & & & & & & & & & $\mathrm{X}$ \\
\hline & Diplasia karatifolia Rich. ex Pers. & & & & $x$ & & $\mathrm{X}$ & & & \\
\hline & Eleocharis confervoides (Poir.) Steud. & $\mathrm{x}$ & & & & & & $\mathrm{X}$ & & \\
\hline & Eleocharis filiculmis Kunth & $\mathrm{X}$ & & & & & & & & \\
\hline & Eleocharis fluctuans (L.T. Eiten) E.H. Roalson \& C.E. Hinchliff & $\mathrm{x}$ & & & & & & $\mathrm{X}$ & & \\
\hline & Eleocharis minima Kunth & & & & $\mathrm{x}$ & & & & & $\mathrm{X}$ \\
\hline & Eleocharis plicarhachis (Griseb.) Svenson & & & & & & & $\mathrm{X}$ & & \\
\hline & Everardia montana subsp. velutina T.Koyama & & & & $\mathrm{X}$ & & & & & \\
\hline & Fimbristylis aestivalis Vahl & $\mathrm{x}$ & $\mathrm{x}$ & & & & & & & \\
\hline & Fimbristylis autumnalis (L.) Roem. \& Schult. & $\mathrm{x}$ & & & & & & $\mathrm{X}$ & & \\
\hline & Fimbristylis dichotoma (L.) Vahl & & & & & & $\mathrm{X}$ & & & \\
\hline & Fimbristylis miliacea (L.) Vahl & & $\mathrm{X}$ & & & & & & & \\
\hline & Fimbristylis vahlii (Lam.) Link & & $\mathrm{X}$ & & & & & & & \\
\hline & Hypolytrum longifolium (Rich.) Nees & & & & $\mathrm{X}$ & & $\mathrm{X}$ & $\mathrm{X}$ & $\mathrm{X}$ & \\
\hline & Hypolytrum rubescens Huber ex C.B.Clarke & & & & & & & $\mathrm{X}$ & & \\
\hline & Hypolytrum strictum Poepp. \& Kunth & & & & & & & & $\mathrm{X}$ & $\mathrm{X}$ \\
\hline & Lagenocarpus guianensis Lindl. ex Nees & & & & & & & & & $\mathrm{X}$ \\
\hline & Lagenocarpus rigidus Nees & & & & & & & $\mathrm{X}$ & & \\
\hline & Lagenocarpus sabanensis Gilly & $\mathrm{X}$ & & & & & & & & \\
\hline & Rhynchospora amazonica Poepp. \& Kunth & & & & $\mathrm{X}$ & $\mathrm{X}$ & & & $\mathrm{X}$ & \\
\hline & Rhynchospora cariciformis Nees & & & & & & $\mathrm{X}$ & & & \\
\hline & Rhynchospora cephalotes (L.) Vahl & & & & & & $\mathrm{X}$ & $\mathrm{X}$ & & \\
\hline & Rhynchospora divaricata (Ham.) M.T.Strong & & & & $\mathrm{x}$ & & & & & \\
\hline & Rhynchospora exaltata Kunth & $\mathrm{X}$ & & & & & & & & \\
\hline & Rhynchospora holoschoenoides (Rich.) Herter & & & & & & & $\mathrm{X}$ & & $\mathrm{X}$ \\
\hline & Rhynchospora paraensis Schrad. ex Kunth & & & & & & & & $\mathrm{x}$ & \\
\hline & Rhynchospora reptans (Rich.) Kük. & & & & & $\mathrm{X}$ & & & $\mathrm{X}$ & $\mathrm{x}$ \\
\hline & Rhynchospora splendens Lindm. & & & & & & & $\mathrm{X}$ & & \\
\hline & Scleria cyperina Willd. ex Kunth & & & & & & $\mathrm{X}$ & & $\mathrm{X}$ & \\
\hline
\end{tabular}




\begin{tabular}{|c|c|c|c|c|c|c|c|c|c|c|}
\hline & Scleria gaertneri Raddi & & & & $\mathrm{X}$ & & & & & \\
\hline & Scleria microcarpa Nees ex Kunth & & $\mathrm{X}$ & & & & & & $\mathrm{x}$ & $\mathrm{X}$ \\
\hline & Scleria secans (L.) Urb. & & & & $\mathrm{X}$ & $\mathrm{X}$ & $\mathrm{X}$ & $\mathrm{X}$ & $\mathrm{x}$ & $\mathrm{X}$ \\
\hline & Eriocaulon sp. & & & & & & & $\mathrm{X}$ & & \\
\hline Eriocaulaceae & Paepalanthus aff. caldensis Malme & & & & & $\mathrm{X}$ & & & $\mathrm{x}$ & $\mathrm{X}$ \\
\hline & Tonina fluvitialis Aubl. & & & & & & & & $\mathrm{x}$ & $\mathrm{X}$ \\
\hline Funhorbiaç & Caperonia castaneifolia (L.) A.St.-Hil. & & & $\mathrm{X}$ & & & & & & \\
\hline Luprivisiaceate & Croton trinitatis Millsp. & $\mathrm{X}$ & & & & & & & & \\
\hline & Clitoria falcata Lam. & & & $\mathrm{X}$ & & & & & & \\
\hline Eahacose & Cymbosema roseum Benth. & & & $\mathrm{X}$ & & & & & & \\
\hline raodecele & Vigna lasiocarpa (Mart.ex Benth.) Verdc. & & & $\mathrm{x}$ & & & & & & \\
\hline & Zornia latifolia Sm. & & & & & & & & & $\mathrm{X}$ \\
\hline & Coutoubea ramosa Aubl. & & & & & & & $\mathrm{X}$ & & \\
\hline Gentianaceae & Irlbachia poeppigii (Griseb.) L.Cobb \& Maas & & & & $\mathrm{X}$ & & $\mathrm{X}$ & & & \\
\hline & Irlbachia pumila (Benth.) Maguire & & & & & & & & $\mathrm{x}$ & $\mathrm{X}$ \\
\hline Holiconiasone & Heliconia julianii Barreiros & & & & & $\mathrm{x}$ & & & & \\
\hline Hentilinaceat & Heliconia psittacorum L.f. & & & & & $\mathrm{X}$ & & & & \\
\hline Hydrocharitaceae & Apalanthe granatensis (Bonpl.) Planch. & $\mathrm{X}$ & & & & & $\mathrm{x}$ & & & \\
\hline Humenophullacean & Trichomanes hostmannianum (Klotzsch) Kunze & & & & & & $\mathrm{X}$ & & & \\
\hline 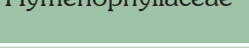 & Trichomanes martiusii C.Presl & & & & $\mathrm{X}$ & & & & & \\
\hline & Hyptis atrorubens Poit. & & & & $\mathrm{X}$ & & & & & \\
\hline Lamiaceae & Hyptis parkeri Benth. & $\mathrm{X}$ & $\mathrm{X}$ & & & & & & $\mathrm{x}$ & \\
\hline & Hyptis sp. & & $\mathrm{X}$ & & & & & & & \\
\hline & Utricularia foliosa L. & & & $\mathrm{X}$ & & & & & & \\
\hline Jontibyl-viา & Utricularia gibba L. & & & & $\mathrm{X}$ & & & & & \\
\hline Lentiouraniacede & Utricularia nervosa G.Weber ex Benj. & & & & & & & & & $\mathrm{X}$ \\
\hline & Utricularia subulata L. & & & & $\mathrm{X}$ & & & & & \\
\hline I indorniacea & Lindernia crustacea (L.) F.Muell. & $\mathrm{X}$ & & & & & & & & $\mathrm{X}$ \\
\hline Lintuentilaceat & Lindernia diffusa (L.) Wettst. & & & & & $\mathrm{X}$ & & & $\mathrm{X}$ & \\
\hline Malvaceae & Hibiscus furcellatus Desr. & & & & & & & & $\mathrm{X}$ & $\mathrm{X}$ \\
\hline & Goeppertia cannoides (Nicolson, Steyerm. \& Sivad.) Borchs. \& S.Suárez & & & & & & $\mathrm{X}$ & & & \\
\hline & Goeppertia microcephala (Poepp. \& Endl.) Borchs. \& S.Suárez & & & & & & & & & $\mathrm{X}$ \\
\hline & Ischnosiphon cannoideus L.Andersson & & & & $\mathrm{X}$ & & & & & \\
\hline & Ischnosiphon crassispicus L.Andersson & & & & $\mathrm{X}$ & & & & & \\
\hline Marantaceae & Ischnosiphon gracilis (Rudge) Körn. & & & & & & & & & $\mathrm{X}$ \\
\hline intantacede & Ischnosiphon longiflorus K.Schum. & & & & & $\mathrm{X}$ & & & $\mathrm{x}$ & $\mathrm{X}$ \\
\hline & Ischnosiphon polyphyllus (Poepp. \& Endl.) Körn. & & & & & & & & & $\mathrm{X}$ \\
\hline & Ischnosiphon puberulus Loes. & & & & & $\mathrm{X}$ & & $\mathrm{X}$ & & $\mathrm{X}$ \\
\hline & Monotagma breviscapum Hagberg \& R.Erikss. & & & & & & & & & $\mathrm{X}$ \\
\hline & Monotagma tuberosum Hagberg \& R. Erikss & & & & & & $\mathrm{X}$ & & & \\
\hline Mayacaceae & Mayaca longipes Mart. ex Seub. & & & & & & & $\mathrm{X}$ & & \\
\hline Molactomatacea & Aciotis acuminifolia (Mart. ex DC.) Triana & & & & & & & & & $\mathrm{X}$ \\
\hline e & Aciotis ornata (Miq.) Gleason & & & & $\mathrm{X}$ & & & & & \\
\hline & Nymphaea aff. oxypetala Planch. & $\mathrm{X}$ & & & & & & & $x$ & $\mathrm{X}$ \\
\hline Numphaอา & Nymphaea amazonum Mart. \& Zucc. & & & & & & $\mathrm{x}$ & & & \\
\hline ivgmpnaedcede & Nymphaea cf. belophylla Trickett & & & & & $\mathrm{X}$ & & & $\mathrm{x}$ & $\mathrm{X}$ \\
\hline & Nymphaea gardneriana Planch. & & & & & & $\mathrm{X}$ & & & \\
\hline Ochnaceae & Sauvagesia erecta L. & & & & & & & & & $\mathrm{X}$ \\
\hline
\end{tabular}




\begin{tabular}{|c|c|c|c|c|c|c|c|c|c|c|}
\hline \multirow{4}{*}{ Onagraceae } & Ludwigia erecta (L.) H.Hara & $\mathrm{X}$ & $\mathrm{X}$ & & & & & & & \\
\hline & Ludwigia hyssopifolia (G.Don) Exell & & & & $\mathrm{X}$ & & & & & \\
\hline & Ludwigia octovalvis (Jacq.) P.H.Raven & & & $\mathrm{X}$ & & & & & & \\
\hline & Ludwigia palustris (L.) Elliott & $\mathrm{X}$ & & & & & & & & \\
\hline Orchidaceae & Palmorchis sobralioides Barb.Rodr. & & & & & & & & $\mathrm{X}$ & \\
\hline \multirow{3}{*}{ Passifloraceae } & Passiflora aff. pohlii Mast. & & & $\mathrm{X}$ & & & & & & \\
\hline & Passiflora glandulosa Cav. & & & & & $\mathrm{x}$ & & & & \\
\hline & Passiflora misera Kunth & & & $\mathrm{X}$ & & & & & & \\
\hline \multirow{33}{*}{ Poaceae } & Acroceras zizanioides (Kunth) Dandy & & $\mathrm{X}$ & & & & & & & \\
\hline & Andropogon bicornis L. & & & & & $\mathrm{X}$ & & & & \\
\hline & Andropogon selloanus (Hack.) Hack. & & & & & & & & & $\mathrm{X}$ \\
\hline & Axonopus fissifolius (Raddi) Kuhlm. & & & & & & & $\mathrm{X}$ & & \\
\hline & Axonopus leptostachyus (Flüggé) Hitchc. & & & & & $\mathrm{x}$ & & & & $\mathrm{X}$ \\
\hline & Axonopus purpusii (Mez) Chase & & & & & $\mathrm{X}$ & & & & $\mathrm{X}$ \\
\hline & Digitaria ciliaris (Retz.) Koeler & & $\mathrm{X}$ & & & & & & & \\
\hline & Echinochloa polystachya (Kunth) Hitchc. & & $\mathrm{X}$ & & & & & & & \\
\hline & Eragrostis maypurensis (Kunth) Steud. & & & & & & & & $\mathrm{X}$ & $\mathrm{X}$ \\
\hline & Guadua aff. ciliata Londoño \& Davidse & & & & $\mathrm{X}$ & & & & & \\
\hline & Hymenachne amplexicaulis (Rudge) Nees & & & $\mathrm{x}$ & $\mathrm{X}$ & & & & & \\
\hline & Olyra latifolia $\mathrm{L}$. & & & & & $\mathrm{x}$ & & & $\mathrm{X}$ & \\
\hline & Olyra longifolia Kunth & & & & $\mathrm{X}$ & & $\mathrm{X}$ & $\mathrm{X}$ & & \\
\hline & Oryza glumaepatula Steud. & & & $\mathrm{x}$ & & & & & & \\
\hline & Oryza rufipogon Griff. & & & & $\mathrm{X}$ & & $\mathrm{x}$ & & & \\
\hline & Panicum aquaticum Poir. & & & & & & & & $\mathrm{X}$ & \\
\hline & Panicum dichotomiflorum Michx. & $\mathrm{X}$ & & & & & $\mathrm{X}$ & & & \\
\hline & Pariana radiciflora Sagot ex Döll & & & & & $\mathrm{x}$ & & & $\mathrm{X}$ & $\mathrm{X}$ \\
\hline & Paspalum conjugatum P.J.Bergius & & & & & $\mathrm{x}$ & & & & \\
\hline & Paspalum decumbens Sw. & & & & & & & & & $\mathrm{X}$ \\
\hline & Paspalum densum Poir & & & & & & & & $\mathrm{X}$ & $\mathrm{X}$ \\
\hline & Paspalum multicaule Poir. & & $\mathrm{X}$ & & & & & & & \\
\hline & Paspalum pulchellum Kunth & & $\mathrm{X}$ & & & & & & & \\
\hline & Paspalum repens P.J.Bergius & & $\mathrm{X}$ & $\mathrm{x}$ & & & & & & \\
\hline & Paspalum scrobiculatum L. & & & & & & & & $\mathrm{X}$ & \\
\hline & Paspalum sp. & & & & & & & & $\mathrm{X}$ & \\
\hline & Reimarochloa brasiliensis (Spreng.) Hitchc. & $\mathrm{X}$ & $\mathrm{X}$ & & & & & & & \\
\hline & Rugoloa hylaeica (Mez) Zuloaga & & & & & & & & $\mathrm{X}$ & \\
\hline & Rugoloa pilosa (Sw.) Zuloaga & & & & & $\mathrm{X}$ & & & & $\mathrm{X}$ \\
\hline & Steinchisma laxum (Sw.) Zuloaga & & & & & $\mathrm{X}$ & & & $\mathrm{X}$ & $\mathrm{X}$ \\
\hline & Trichanthecium cyanescens (Nees ex Trin.) Zuloaga \& Morrone & & & & & & & $\mathrm{X}$ & & \\
\hline & Trichanthecium micranthum (Kunth) Zuloaga \& Morrone & & & & & & & $\mathrm{X}$ & & \\
\hline & Urochloa mutica (ForssK.) Ngeryen & & & & & $\mathrm{x}$ & & & & $\mathrm{X}$ \\
\hline Podostemaceae & Oserya perpusilla (Went) P.Royen & & & & $\mathrm{X}$ & & & & & \\
\hline Pontederiaceae & Eichhornia crassipes (Mart.) Solms & & & $\mathrm{X}$ & & & & & & \\
\hline Pteridaceae & Ceratopteris pteridoides (Hook.) Hieron. & & & $\mathrm{x}$ & & & & & & \\
\hline \multirow{3}{*}{ Rapateaceae } & Duckea squarrosa (Willd. ex Link) Maguire & & & & & & & $\mathrm{X}$ & & \\
\hline & Saxofridericia aculeata Körn & & & & & & $\mathrm{x}$ & & & \\
\hline & Spathanthus unilateralis (Rudge) Desv. & & & & & & & $\mathrm{X}$ & & $\mathrm{X}$ \\
\hline \multirow{2}{*}{ Rubiaceae } & Borreria capitata (Ruiz \& Pav.) DC. & & & & & $\mathrm{x}$ & & & $\mathrm{X}$ & $\mathrm{X}$ \\
\hline & Spermacoce reflexa (J.H.Kirkbr.) Govaerts & & & & & & & & & $\mathrm{X}$ \\
\hline
\end{tabular}




\begin{tabular}{|c|c|c|c|c|c|c|c|}
\hline Salviniaceae & Salvinia auriculata Aubl. & & & $\mathrm{X}$ & & & \\
\hline Sellaginellaceae & Selaginella conduplicata Spring & & & & $\mathrm{x}$ & & \\
\hline \multirow{2}{*}{ Solanaceae } & Physalis angulata $\mathrm{L}$. & $\mathrm{X}$ & & & & & \\
\hline & Schwenckia grandiflora Benth. & & & $\mathrm{X}$ & & & \\
\hline Thurniaceae & Thurnia sphaerocephala (Rudge) Hook.f. & & & & & $\mathrm{X}$ & \\
\hline Turneraceae & Piriqueta cistoides (L.) Griseb. subsp. cistoides & & $\mathrm{X}$ & & & & \\
\hline Vitaceae & Cissus erosa Rich. & & & $\mathrm{X}$ & $\mathrm{X}$ & & $\mathrm{X}$ \\
\hline Xyridaceae & Xyris jupicai Rich. & & & & & & $\mathrm{x}$ \\
\hline
\end{tabular}

As quatro áreas que estão dentro de UCs: Reserva de Desenvolvimento Sustentável (RDS) do Uatumã (rios Uatumã e Abacate), Parque Nacional (PARNA) do Jaú, e PARNA de Anavilhanas apresentaram, juntas, 86 espécies, ou seja, apenas 49,2\% das espécies de igapó inventariadas, mostrando que a maior parte das espécies de herbáceas aquáticas encontra-se em áreas fora das unidades de conservação inventariadas. Embora dentre as espécies levantadas não tenha sido registrada nenhuma espécie da flora ameaçada de extinção, foram encontradas 28 espécies com ocorrência apenas para a bacia Amazônica, sendo que apenas 10 dessas espécies ocorreram dentro das UCs inventariadas. Em princípio, pode não parecer um fato preocupante, mas, tendo em vista que há cerca de duas mil espécies em alguma categoria de ameaça, e que até o momento foram avaliadas apenas 6 mil das mais de 46 mil existentes (MMA 2014, CNCFlora 2018), o número de espécies ameaçadas pode estar bastante subestimado e encobrindo uma situação provavelmente bastante crítica. Dentre as microbacias do rio Negro, 12 foram consideradas prioritárias para conservação na categoria extremante alta, uma como muito alta e 33 como alta (Loyola et al. 2014), devido à pressão que recebem com relação ao desmatamento, à instalação de grandes empreendimentos e ao uso intensivo da terra.

A análise de escalonamento multidimensional resultou em uma ordenação das espécies em duas dimensões, com stress de 0,23 (Figura 2). É evidente o agrupamento de três pontos do rio Negro, Santa Isabel do Rio Negro, arquipélago Mariuá e Barcelos, os quais também são geograficamente próximos (Figura 1). Houve também a aproximação entre os pontos referentes aos inventários dos rios Aracá e Abacate, embora esses rios pertençam a bacias diferentes; e dos rios Uatumã e Cuiuni, ficando os inventários dos rios Jaú e Negro (PARNA de Anavilhanas) bastante afastados dos demais em relação à composição florística. O índice de Jaccard confirmou a baixa similaridade de espécies entre as áreas de igapó inventariadas (Figura 3). Apenas as áreas às margens do rio Negro nas áreas de Barcelos, Santa Isabel do Rio Negro e arquipélago Mariuá mostraram similaridade acima de $30 \%$ entre si (Figura 3). Lopes et al. (2014) também encontraram essa separação do rio Jaú no NMDS em relação aos outros igapós por eles estudados, justificando essa separação pelas particularidades da geomorfologia daquele rio e sua bacia. De acordo com esses autores, "o baixo rio Jaú possui poucos lagos e meandros, segue um canal rochoso e encaixado, e assim tem poucos habitat de menor correnteza que favorecem a presença de várias espécies de plantas aquáticas" (Junk \& Piedade 1997). Além disso, o solo rochoso e a elevada correnteza seriam fortes limitantes para o estabelecimento de muitas espécies de plantas aquáticas (Sculthorpe 1985). Já o igapó do PARNA de Anavilhanas tem a singularidade de o baixo Negro receber nutrientes do rio Branco, apresentando sedimentos finos, que são ligeiramente mais férteis do que aqueles localizados rio acima (Junk et al. 2011). Montero et al. (2014) mostraram que, para as espécies arbóreas, houve $62 \%$ de similaridade florística nos igapós do rio Negro, valor bastante superior do encontrado no presente estudo para herbáceas aquáticas (30\%). 


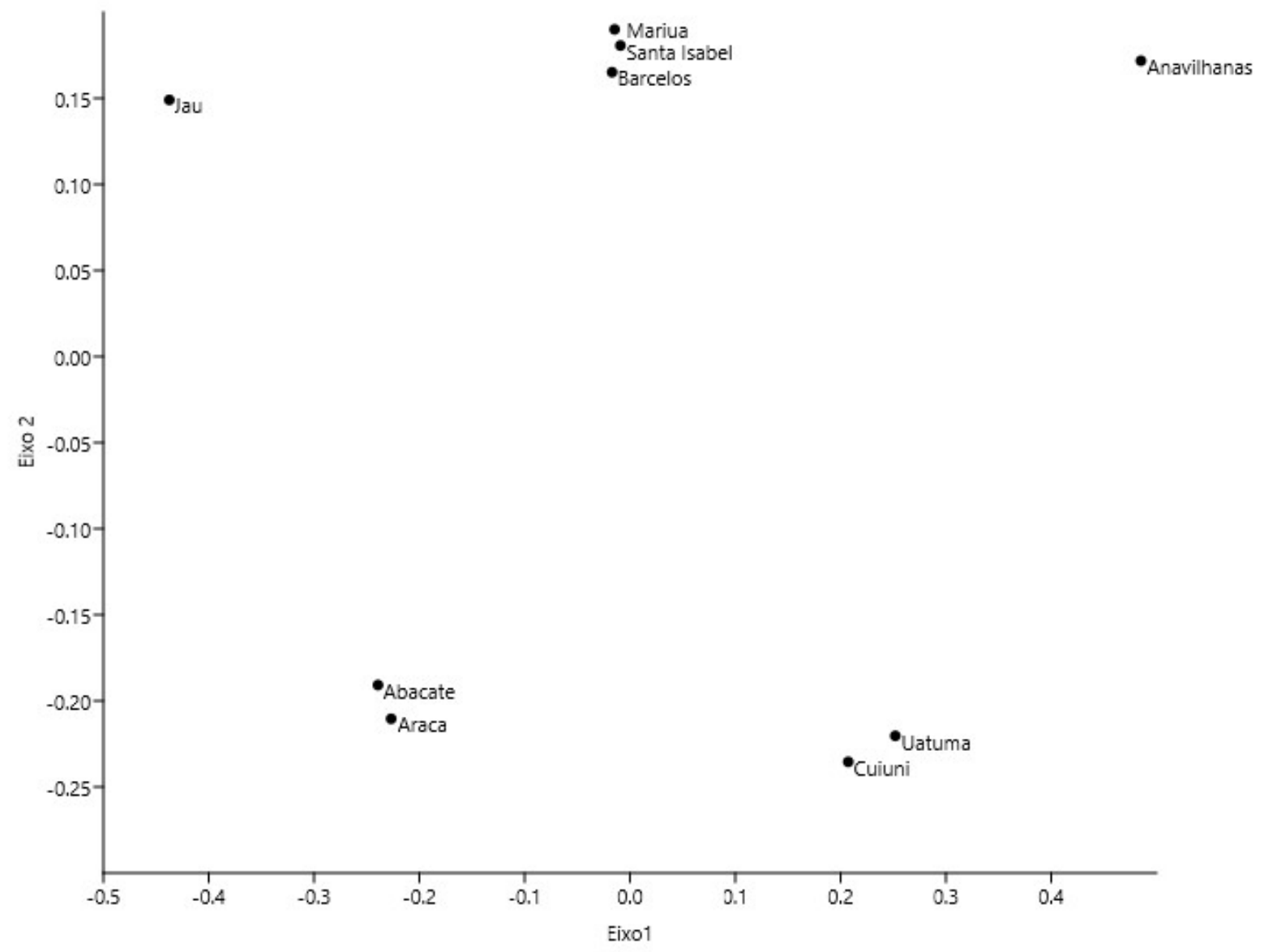

Figura 2 - Ordenação em dois eixos do NMDS das parcelas de herbáceas aquáticas dos igapós de água preta dos rios Cuiuni, Uatumã, Negro (PARNA de Anavilhanas), Jaú, Negro (arquipélago de Mariuá), Abacate, Aracá, Negro (Barcelos), Negro (Santa Isabel do Rio Negro) na Amazônia brasileira.

Figure 2 - Ordination on two axes of NMDS of aquatic herbaceous plots in black water rivers "igapós" of rivers Cuiuni, Uatumã, Negro (PARNA de Anavilhanas), Jaú, Negro (arquipélago de Mariuá), Abacate, Aracá, Negro (Barcelos), Negro (Santa Isabel do Rio Negro) in the brazilian Amazon. 


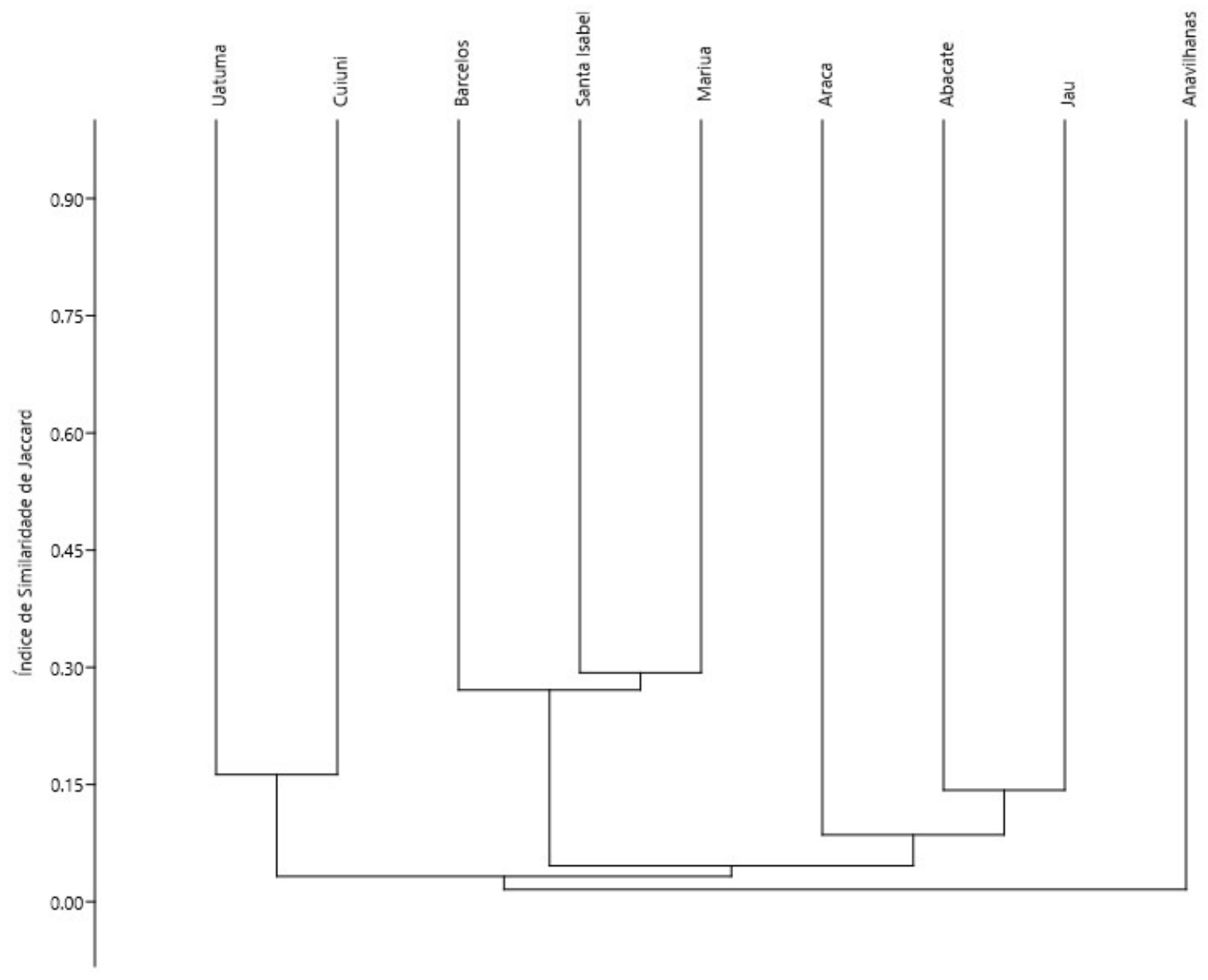

Figura 3 - Índice de similaridade florística de herbáceas aquáticas usando a distância de Jaccard das nove áreas de igapós inventariadas na bacia Amazônica.

Figure 3 - Jaccard similarity index by aquatic herbaceous plants of the nine areas of black-water "igapós" inventoried in Amazon basin. 
De acordo com o índice de Simpson, a diversidade alfa de herbáceas aquáticas foi maior no rio Negro, no sítio de Santa Isabel do Rio Negro (Tabela 4). A diversidade Beta Global pelo índice de Whittaker foi calculada em 5,14 para os igapós de água preta da Amazônia. As áreas que apresentaram menor diversidade $\beta$, ou seja, onde a substituição de espécies foi menor quando comparadas, foram os igapós do Negro nas áreas do arquipélago Mariuá e Santa Isabel do Rio Negro, com 0,55 (Tabela 5). Altos valores de diversidade beta e baixos valores de diversidade alfa também foram encontrados por Montero et al. (2014) para espécies arbóreas nos igapós do rio Negro, confirmando a grande variação florística entre os sítios inventariados, tanto para as espécies arbóreas quanto para as herbáceas aquáticas.

Tabela 4 - Diversidade beta de herbáceas aquáticas pelo índice de Whittaker para os igapós de água preta inventariados na bacia Amazônica.

Table 4 - Beta diversity of aquatic herbaceous species by Whittaker index for black-water floodplains "igapós" inventoried in the Amazon basin.

\begin{tabular}{|c|c|c|c|c|c|c|c|c|}
\hline & Cuiuni & Abacate & Aracá & $\begin{array}{c}\text { Negro } \\
\text { (Barcelos) }\end{array}$ & $\begin{array}{l}\text { Negro } \\
\text { (Santa } \\
\text { Isabel } \\
\text { do Rio } \\
\text { Negro) }\end{array}$ & $\begin{array}{c}\text { Negro } \\
\text { (arquipélago } \\
\text { de } \\
\text { Mariuá) }\end{array}$ & Jaú & $\begin{array}{c}\text { Negro } \\
\text { (PARNA de } \\
\text { Anavilhanas) }\end{array}$ \\
\hline Abacate & 0,87 & & & & & & & \\
\hline Aracá & 0,88 & 0,80 & & & & & & \\
\hline Negro (Barcelos) & 0,89 & 0,86 & 0,93 & & & & & \\
\hline $\begin{array}{l}\text { Negro (Santa Isabel do Rio } \\
\text { Negro) }\end{array}$ & 0,92 & 0,94 & 0,87 & 0,58 & & & & \\
\hline $\begin{array}{l}\text { Negro (arquipélago de } \\
\text { Mariuá) }\end{array}$ & 0,96 & 0,92 & 0,93 & 0,57 & 0,55 & & & \\
\hline Jaú & 1,00 & 0,75 & 0,88 & 0,89 & 0,94 & 0,92 & & \\
\hline $\begin{array}{l}\text { Negro (PARNA de } \\
\text { Anavilhanas) }\end{array}$ & 0,96 & 1,00 & 1,00 & 0,96 & 1,00 & 0,96 & 0,96 & \\
\hline Uatumã & 0,72 & 0,96 & 1,00 & 0,90 & 0,95 & 0,96 & 0,96 & 0,92 \\
\hline
\end{tabular}

\section{Conclusões}

Embora os valores de diversidade alfa para herbáceas aquáticas em igapós de água preta tenham sido parecidos entre as áreas, a diversidade beta foi alta entre os sítios inventariados, mostrando que a flora de cada área é bastante diferenciada. Além disso, as UCs inventariadas contiveram, aproximadamente, 49,4\% do total das espécies de herbáceas aquáticas amostradas.

Considerando que no Brasil as unidades de conservação são as mais importantes formas para a proteção de espécies, e que a flora dos igapós possui alta diversidade beta, acreditamos que a criação e a manutenção de UCs que englobem diferentes regiões da bacia do rio Negro e tributários são essenciais para a proteção de um maior número de espécies de herbáceas aquáticas $e$, consequentemente, da sua biota associada. 


\section{Agradecimentos}

Este estudo é parte do PELD MAUA (CNPq/FAPEAM) e contou com o financiamento do Conselho Nacional de Desenvolvimento Científico e Tecnológico (CNPq/PELD-MAUA fase II), n. 441590/2016-0; Instituto Chico Mendes de Conservação da Biodiversidade, Pronex Tipologias Alagáveis Amazônicas (2007-2011), Edital universal CNPq 2007-2009; 2009-2011; 2011-2013, INCT ADAPTA (CNPq/FAPEAM), FIXAM/FAPEAM (017/2914). Os autores agradecem pela bolsa PNPD concedida à A.L. pela Coordenação de Aperfeiçoamento de Pessoal de Nível Superior Brasil (CAPES), Código de Financiamento 001, e pela bolsa AT-FIXAM concedida pela FAPEAM a I.N.S. e A.B.F. Agradecemos à Sammya Agra D'Angelo, Naara Ferreira da Silva, Pauline de Oliveira Pantoja, pelo auxílio na coleta e organização dos dados. A Valdeney de Araújo Azevedo, Mário Luis Picanço Marinho e Conceição Lucia Costa, pela ajuda nas coletas de campo; e à Vali Joana Pott (UFMS) e José Ferreira Ramos (INPA), agradecemos pelo auxílio nas identificações.

\section{Referências bibliográficas}

APG IV. 2016. An update of the Angiosperm Phylogeny Group classification for the orders and families of flowering plants: APG IV. Botanical Journal of the Linnean Society, 181: 1-20.

Arraut, E.M.; Marmontel, M.; Mantovani, J.E.; Novo, E.M.L.; Macdonald, D.W. \& Kenward, R.E. 2010. The lesser of two evils: seasonal migrations of Amazonian manatees in the Western Amazon. Journal of Zoology, 280(3): 246-256.

Barendregt, A. \& Bio, A.M.F. 2003. Relevant variables to predict macrophytes communities in running waters. Ecological Modelling, 160: 205-217.

Best, R.C. 1984. The aquatic mammals and reptiles of the amazon, p. 372-412. In: Sioli, H. (ed.). The amazon limnology and landscape ecology of a mighty tropical river and its basins. Dr. W. Junk Publisher, 800p.

Brasil, 2000. Lei n 9.985 de 18 de julho de 2000. SNUC - Sistema Nacional de Unidades de Conservação. Ministério do Meio Ambiente. Diário Oficial da União. <http:/www.mma.gov.br/port/conoma/legiabre. cfm?codlegi=322 > . (Acesso em 20/11/2017).

Cancian, L.F.; Camargo, A.F.M. \& Silva, G.H.G. 2009. Crescimento de Pistia stratiotes em diferentes condições de temperatura e fotoperíodo. Acta Botanica Brasilica, 23(2): 552-557.

CNCFlora 2018. Centro Nacional de Conservação da Flora. <http://cncflora.jbrj.gov.br/portal>. (Acesso em 10/01/2018).

Crema, L.C. 2017. Caracterização de igapós de águas claras e pretas e suas disponibilidades alimentares para o peixe-boi-da-Amazônia (Trichechus inunguis). Tese (Doutorado em Biologia de Água Doce e Pesca Interior). Instituto Nacional de Pesquisas da Amazônia. 121p.

Crema, L.C.; Silva, V.M.F. \& Piedade, M.T.F. 2019. Riverine people's knowledge of the endangered amazonian manatee Trichechus Inunguis in contrasting protected areas. Oryx, 831: 1-10.

Esteves, F.A. 1998. Fundamentos de limnologia. 2 ed. Interciência. 575p.

Ferreira, L.V. 2000. Effects of flooding duration on species richness, floristic composition and forest structure in river margin habitat in Amazonian blackwater floodplain forest: implications for future design of protected areas. Biodiversity and Conservation, 9(1): 1-14.

Fernandes, R.; Gomes, L.C.; Pelicice, F.M. \& Agostinho, A.A. 2009. Temporal organization of fish assemblages in floodplain lagoons: the role of hydrological connectivity. Environmental Biology of Fishes, 85(2): 99-108.

Fittkau, E.J.; Junk, W.J.; Klinge, H. \& Sioli, H. 1975. Substrate and vegetation in the Amazonan region, p. 73-90. In: Cramer. J. (ed.). Vegetation und substrat. Beriohto dor internationalen Symposien der Internationalen Vereinigung für Vegetationskunde. 
Furch, K. \& Junk, W.J. 1992. Nutrient dynamics of submersed decomposing Amazonian herbaceus plant species Paspalum fasciculatum and Echinochloa Polystachya. Revue d'Hydrobiologie Tropicale, 25(2): 75-85.

Furch, K. \& Junk, W.J. 1997. Chemical composition, food value and decomposition of herbaceous plants leaves and leaf litter of the floodplain forest, p. 187-203. In: Junk, W.J. (ed.). The Central Amazon Floodplain: ecology of a pulsing system. Ecological Studies. Springer-Verlag.126. 528p.

GBIF - Global Biodiversity Information Facility. <http://www.gbif.org/species/5284517>. (Acesso em 30/11/2017).

Hammer, $\varnothing$; Harper, D.A.T. \& Ryan, P.D. 2001. PAST: paleontological statistics software package for education and data analysis. Palaeontologia Electronica.

Hassler, M.L. 2005. A importância das unidades de conservação no Brasil. Sociedade e Natureza, 17(33): 79-89.

Henry-Silva, G.G. \& Camargo, A.F.M. 2005. Interações ecologicas entre as macrófitas aquáticas flutuantes Eichhornia crassipes e Pistia stratiotes. Hoehnea, 32(3): 445-452.

ICMBio 2018. Ministério do Meio Ambiente. Unidades de Conservação. < http://www.icmbio.gov.br/portal/ unidadesdeconservacao/biomas-brasileiros > . (Acesso em 10/01/2018).

Instituto Chico Mendes de Conservação da Biodiversidade. Painel Corportativo. <hhttp://qvicmbio.gov.br/ QvAJAXZfc/opendoc2.htm?document=painel_corporativo_6476.qvw\&host $=$ Local\&anonymous $=$ true $>$. (Acesso em 21/09/2018).

Junk. W.J. 1980. Áreas inundáveis: um desafio para limnologia. Acta Amazonica, 10(4): 775-795.

Junk, W.J. 1986. Aquatic plants of the Amazon system, p. 319-337. In: Davies, W.B. (ed.). The Ecology of River Systems. Dr. W. Junk Publishers, 793p.

Junk, W.J.; Bayley, P.B. \& Sparks, R.E. 1989. The flood pulse concept in river-floodplain systems. In: Dodge, D.P. (ed.). Proceedings of the International Large River Symposium (LARS). Canadian Journal of Fisheries and Aquatic Sciences, 106: 110-127.

Junk, W.J. \& Furch, K. 1991. Nutrient dynamics in Amazonian floodplains: decomposition of herbaceous plants in aquatic and terrestrial environments. Internationale Vereinigung für theoretische und angewandte Limnologie: Verhandlungen, 24(4): 2080-2084.

Junk, W.J. \& Piedade, M.T.F. 1993. Herbaceous plants of the Amazon floodplain near Manaus: Species diversity and adaptations to the flood pulse. Amazoniana, 12(3/4): 467-484.

Junk, W.J. \& Piedade, M.T.F. 1997. Plant life in the floodplain with special reference to herbaceous plants, p. 147-185. In: Junk, W.J. (ed.). The Central Amazon Floodplain: ecology of a pulsing system. Ecological Studies. Springer-Verlag.126. 528p.

Junk, W.J. \& Silva, V.M.F. 1997. Mammals, reptiles and amphibians, p. 409-417. In: Junk, W.J. (ed.). The Central Amazon Floodplain: ecology of a pulsing system. Ecological Studies. SpringerVerlag.126. 528p.

Junk, W.J; Piedade, M.T.F; Schöngart, J.; Cohn-Haft, M.; Adeney, J.M. \& Wittmann, F. 2011. A Classification of Major Naturally-Occurring Amazonian Lowland Wetlands. Wetlands, 31(4): 623-640.

Junk, W.J.; Piedade, M.T.F.; Schöngart, J. \& Wittmann, F. 2012. A classification of major natural habitats of Amazonian white-water river floodplains (várzeas). Wetlands Ecology and Management, 20(6): 461-475.

Junk, W.J.; Wittmann, F.; Schöngart, J. \& Piedade, M.T.F. 2015. A classification of the major habitats of Amazonian black-water river floodplains and a comparison with their white-water counterparts. Wetlands Ecology and Management, 23(4): 677-693.

Küchler, I.L.; Miekeley, N. \& Forsberg, B.R. 2000. A contribution to the chemical characterization of rivers in the Rio Negro Basin, Brazil. Journal of the Brazilian Chemical Society, 11(3): 286-292. 
Lopes, A.; Wittmann, F; Schöngart, J. \& Piedade, M.T.F. 2014. Herbáceas aquáticas em seis igapós na Amazônia Central: Composição e diversidade de gêneros. Geográfica Acadêmica, 8(1): 5-17.

Lopes, A.; Parolin, P. \& Piedade, M.T.F. 2016. Morphological and physiological traits of aquatic macrophytes respond to water chemistry in the Amazon basin: an example of the genus Montrichardia Crueg (Araceae). Hydrobiologia, 766(1): 1-15.

Lopes, A.; Wittmann, F.; Schöngart, J.; Householder, J.E. \& Piedade, M.T.F. 2017. Modeling of regional-and local-scale distribution of the genus Montrichardia Crueg.(Araceae). Hydrobiologia, 789(1): 45-57.

Loyola, R.; Machado, N.; Vila Nova, D.; Martins, E. \& Martinelli, G. 2014. Áreas prioritárias para conservação e uso sustentável da flora brasileira ameaçada de extinção. Andrea Jakobsson Estúdio. Instituto de Pesquisas Jardim Botânico. 80p.

Manly, B.J. 2004. Multivariate statistical methods: a primer. 3 ed. Chapman and Hall. 214p.

Magurran, A.E. 1988. Ecological diversity and its measurement. Princeton University Press. 179p.

MMA 2014. Ministério do Meio Ambiente. Portaria MMA no 443, de 17 dezembro de 2014. <http:// cncflora.jbrj.gov.br/portal/static/pdf/portaria_mma_443_2014.pdf>. (Acesso em 10/01/2018).

Montero, J.C.; Piedade, M.T.F. \& Wittmann, F. 2014. Floristic variation across $600 \mathrm{~km}$ of inundation forests (Igapó) along the Negro River, Central Amazonia. Hydrobiologia, 729(1): 229-246.

Murphy, K.J. 1988. Aquatic weed problems and their management: a review I. The worldwide scale of the aquatic weed problem. Crop Proctetion, 7(4): 232-248.

Neiff, J.J. \& Neiff, A.S.G. 2003. Connectivity processes as a basis for the management of aquatic plants, p. 39-58. In: Thomaz, S. \& Bini, L.M. (eds.). Ecologia e manejo de macrófitas aquáticas. NupéliaMaringá. Eduem. 341p.

Neves, M.A. 2007. Composição, riqueza e variação espaço temporal de macrófitas aquáticas do lago do trevo município de Boa Vista. Dissertação (Mestrado em Recursos Naturais). Universidade Federal de Roraima. 128p.

Petry, R.; Bayley, P.B. \& Markle, D.F. 2003. Relationships between fish assemblages, macrophytes and environmental gradients in the Amazon River floodplain. Journal of Fish Biology, 63(3): 547-579.

Piedade, M.T.F.; Junk, W.J. \& Long, S.P. 1991. The productivity of the C4 grass Echinochloa polystachya on the Amazon floodplain. Ecology, 72(4): 1456-1463.

Piedade, M.T.F. \& Junk, W.J. 2000. Natural herbaceous plant communities in the Amazon floodplains and their use, p. 269-290. In: Junk, W.J.; Ohly, J.; Piedade, M.T.F. \& Soares, M.G.M. (eds.). The Central Amazon Floodplain: actual use and options for a sustainable management. Backhuys Publishers b.v. 584 p.

Piedade, M.T.F.; Schöngart, J. \& Junk, W.J. 2005. O manejo sustentável das áreas alagáveis da Amazônia Central e as comunidades herbáceas aquáticas. Uakari, 1(1): 29-38.

Piedade, M.T.F.; Junk, W.J.; D’Angelo, S.A.; Wittmann, F.; Schöngart, J. \& Lopes, A. 2010. Aquatic herbaceous plants of the Amazon floodplains: state of the art and research needed. Acta Limnologica Brasiliensia, 22(2): 165-17.

Piedade, M.T.F.; Lopes, A.; Demarchi, L.O.; Wittmann, F.; Schöngart, J.; Junk, W. \& Cruz, J. 2018. Guia de Campo de Herbáceas Aquáticas: várzea amazônica. 1. ed. INPA. 299p.

Prance, G.T. 1979. Notes on the vegetation of Amazonia III. The terminology of Amazonian forest types subject to inundation. Brittonia, 31(1): 26-38.

Prance, G.T. 1980. A terminologia dos tipos de florestas Amazônicas sujeitos à inundação. Acta Amazonica, 10(3): 495-504.

Reflora (Flora do Brasil 2020). Jardim Botânico do Rio de Janeiro. <http://floradobrasil.jbrj.gov.br/>. (Acesso em 22/11/2017). 
SEMA 2018 - Unidades de Conservação. <http://meioambiente.am.gov.br/unidade-de-conservacao/>. (Acesso em 10/01/2018).

Sioli, H. 1984. The Amazon and its main affluents: Hydrography, morphology of the river courses, and river types, p. 127-166. In: Sioli, H. (ed.). The Amazon - limnology and landscape ecology of a mighty tropical river and its basin. Dr. W. Junk Publisher, 800p.

Sioli, H. 1985. Amazônia: fundamentos da ecologia da maior região de florestas tropicais. Vozes Ltda. 69p.

Simões Filho, F.S.; Turcq, B.; Carneiro Filho, A. \& Souza, A.G. 1997. Registros sedimentares de lagos e brejos dos campos de Roraima: implicações paleoambientais ao longo do Holoceno, p. 295-305. In: Barbosa, R.I.; Ferreira, E.J.G. \& Castellón, E.G. (eds.). Homem, ambiente e ecologia no estado de Roraima. INPA. $613 p$.

Sneath, P.H.A. \& Sokal, R.R. 1973. Numerical taxonomy. The principles and practice of numerical classification. Freeman. 573p.

Sculthorpe, C.D. 1985. The biology of aquatic vascular plants. Koeltz Scientific Books, 610p.

Thomaz, S.M. 2002. Fatores ecológicos associados à colonização e ao desenvolvimento de macrófitas aquáticas e desafios de manejo. Planta Daninha, 20(1): 21-33.

Tropicos.org. Misouri Botanical Garden. <http://www.tropicos.org/ReferenceSearch.aspx>. (Acesso em 30/11/2017).

Whittaker, R.H. 1960. Vegetation of the Siskiyou Mountains, Oregon and California. Ecological Monographs, 30(3): 279-338.

Biodiversidade Brasileira - BioBrasil.

Número temático: Diagnóstico e manejo de áreas úmidas em áreas protegidas

$$
\text { n. 2, } 2019
$$

http://www.icmbio.gov.br/revistaeletronica/index.php/BioBR

Biodiversidade Brasileira é uma publicação eletrônica científica do Instituto Chico Mendes de Conservação da Biodiversidade (ICMBio) que tem como objetivo fomentar a discussão e a disseminação de experiências em conservação e manejo, com foco em unidades de conservação e espécies ameaçadas.

ISSN: 2236-2886 